PROCEEDINGS OF THE

AMERICAN MATHEMATICAL SOCIETY

Volume 130, Number 7 , Pages 2025-2030

S 0002-9939(01)06253-0

Article electronically published on December 27, 2001

\title{
A FAMILY OF QUADRATIC POLYNOMIAL DIFFERENTIAL SYSTEMS WITH INVARIANT ALGEBRAIC CURVES OF ARBITRARILY HIGH DEGREE WITHOUT RATIONAL FIRST INTEGRALS
}

\author{
COLIN CHRISTOPHER AND JAUME LLIBRE \\ (Communicated by Carmen C. Chicone)
}

\begin{abstract}
We give a class of quadratic systems without rational first integral which contains irreducible algebraic solutions of arbitrarily high degree. The construction gives a negative answer to a conjecture of Lins Neto and others.
\end{abstract}

\section{INTRODUCTION}

By definition a complex planar polynomial differential system or simply a polynomial system is a differential system of the form

$$
\frac{d x}{d t}=\dot{x}=p(x, y), \quad \frac{d y}{d t}=\dot{y}=q(x, y),
$$

where the dependent variables $x$ and $y$ and the independent one (the time) $t$ are complex, and $p$ and $q$ are polynomials in the variables $x$ and $y$ with complex coefficients. We denote by $m=\max \{\operatorname{deg} p, \operatorname{deg} q\}$ the degree of the polynomial system.

Alternatively, we can view (1) as defining a complex foliation with singularities on $\mathbf{C P}^{2}$ of degree $n$. Such a foliation has an invariant line at infinity and conversely any such foliation can be brought to the form (1).

Due to the algebraic structure of a polynomial system there is a close relationship between its integrability (a topological phenomena) and its exact algebraic solutions. Darboux [7] proved that if a polynomial system of degree $m$ has at least $m(m+1) / 2$ invariant algebraic curves, then it has a first integral. Jouanolou [10] (see also [5]) shows that if the number of invariant algebraic curves is at least $[m(m+1) / 2]+2$, then the first integral is rational, and consequently all the trajectories of the vector field are contained in invariant algebraic curves. Prelle and Singer [17, 18] proved that if a polynomial vector field has an elementary or Liouvillian first integral, then this integral can be computed by using the invariant algebraic curves of the system (in fact, for Liouvillian first integrals, a knowledge of the exponential factors of the system is also needed; see [5]).

Received by the editors November 1, 2000 and, in revised form, January 25, 2001.

2000 Mathematics Subject Classification. Primary 34C05, 34A34, 34C14.

Key words and phrases. Rational first integral, invariant algebraic curve, quadratic systems.

(C)2001 American Mathematical Society 
From Jouanolou's result it follows that for a given polynomial system the maximum degree of its invariant algebraic curves is bounded, since either it has a finite number $p<[m(m+1) / 2]+2$ of invariant algebraic curves, or all its trajectories are contained in invariant algebraic curves and the system admits a rational first integral. Thus for each polynomial system there is a natural number $N$ which bounds the degree of all its irreducible invariant algebraic curves. A natural question, going back to Poincaré [16], is to give an effective procedure to find $N$. Partial answers to this question were given by Cerveau and Lins Neto [4], Carnicer [2], and Campillo and Carnicer [1. These results depend on either restricting the nature of the foliation given by (1) or the singularities of the algebraic curves.

Of course, given such a bound, it is then easy to compute the algebraic curves of the system and also describe its elementary or Liouvillian first integrals (modulo any exponential factors); see for instance [13, 3, 15].

Unfortunately, for the class of polynomial systems with fixed degree $m$, there does not exist a uniform upper bound $N(m)$ for $N$ as shown by the system

$$
\dot{x}=r x, \quad \dot{y}=s y,
$$

with $r$ and $s$ positive integers. This system has a rational first integral

$$
H=\frac{y^{r}}{x^{s}},
$$

and hence invariant algebraic curves $x^{s}-h y^{r}=0$ for all $h \in \mathbf{C}$.

However, although $N(m)$ does not exist, maybe there is a number $\tilde{N}(m)$ for which any system with an algebraic curve of degree higher than $\tilde{N}(m)$ will also have an algebraic curve of degree at most $\tilde{N}(m)$. If so the closure of the set of systems (1) of degree $m$ with algebraic curves will be an algebraic subset of the parameter space. At the moment, the most we can say is that this subspace is not dense. Clearly this Noetherian property would be very satisfactory from a theoretical point of view, even if not effective.

Another common suggestion is that there is some number $M(m)$ for which all polynomial systems of degree $m$ with invariant algebraic curves of degree greater than $M(m)$ has a rational first integral (see for instance the open question 2 in [5], or the question at the end of the introduction of [12]).

The purpose of this paper is to give a family of polynomial systems of degree 2 without rational first integrals but with irreducible invariant algebraic curves of arbitrarily high degree. Thus we show that no such function $M(m)$ exists. The question of the function $\tilde{N}(m)$ remains open.

Theorem. We consider the quadratic polynomial differential system

$$
\begin{aligned}
& \dot{x}=x(1-x), \\
& \dot{y}=-\lambda y+A x^{2}+B x y+y^{2},
\end{aligned}
$$

where

$$
\lambda=c-1, \quad A=\frac{a b(c-a)(c-b)}{c^{2}}, \quad B=a+b-1-\frac{2 a b}{c} .
$$

If, for any positive integer $k$, we choose $a=1-k, b \leq a$, and $c$ irrational, then the polynomial system (2) has:

(a) no rational first integrals; 
(b) an irreducible invariant algebraic curve

$$
\left(y-\frac{a b}{c} x\right) F+x(1-x) F^{\prime}=0,
$$

of degree $k$, where $F=F(a, b ; c ; x)$ is the hypergeometric function;

(c) a Darbouxian integrating factor.

The definition of Darbouxian function is given at the end of Section 2, and the precise definition of the hypergeometric function $F(a, b ; c ; x)$ together with the proof of the theorem are provided in Section 3.

Polynomial systems of type (2) were first studied in the work of Françoise, Fronville and Zoladek [8] and later on with more detail in 6]. The family arises naturally when one seeks integrable critical points with $p:-q$ resonance. In fact, the system (2) is linearizable at the origin whenever $\lambda$ is not a non-negative integer. When $\lambda=1$, however, the origin is not a centre and this may account for the neglect of this interesting family until now. Since the solutions are given explicitly, and are very different from other known explicit solutions for quadratic systems, it may be that there are many more interesting properties of these systems and their perturbations to discover.

In the light of this result, we conjecture that there is some number $D(m)$ for which any polynomial system of degree $m$ which has some invariant algebraic curve of degree greater than $D(m)$ has a Darbouxian first integral or Darbouxian integrating factor.

When this note was written we received the paper of Moulin-Ollagnier 14 where he provided another family of polynomial systems of degree 2 without rational first integral, with a Darbouxian first integral or a Darbouxian integrating factor, and with invariant algebraic curves of arbitrarily high degree. However, we feel that our proof is not only much more direct, but also the form of the algebraic curves are given explicitly.

\section{First integrals, integrating factors, INVARIANT ALGEBRAIC CURVES AND DARBOUXIAN FUNCTIONS}

We denote by $\mathbf{C}$ the complex field and by $\mathbf{C}[x, y]$ the ring of polynomials in the variables $x$ and $y$ with coefficients in $\mathbf{C}$.

The vector field $X$ associated to system (1) is defined by

$$
X=p \frac{\partial}{\partial x}+q \frac{\partial}{\partial y} .
$$

The polynomial system (1) is integrable on an open subset $U$ of $\mathbf{C}^{2}$ if there exists a nonconstant analytic function $H: U \rightarrow \mathbf{C}$, called a first integral of the system on $U$, which is constant on all solution curves $(x(t), y(t))$ of system (1) on $U$; i.e. $H(x(t), y(t))=$ constant for all values of $t$ for which the solution $(x(t), y(t))$ is defined on $U$. Clearly $H$ is a first integral of system (1) on $U$ if and only if $X H \equiv 0$ on $U$.

Let $R: U \rightarrow \mathbf{C}$ be an analytic function which is not identically zero on $U$. The function $R$ is an integrating factor of the polynomial system (1) on $U$ if one of the following three equivalent conditions holds:

$$
\frac{\partial(R p)}{\partial x}=-\frac{\partial(R q)}{\partial y}, \quad \operatorname{div}(R p, R q)=0, \quad X R=-R \operatorname{div}(P, Q),
$$


on $U$. As usual the divergence of the vector field $X$ is defined by

$$
\operatorname{div}(X)=\operatorname{div}(p, q)=\frac{\partial p}{\partial x}+\frac{\partial q}{\partial y} .
$$

The first integral $H$ associated to the integrating factor $R$ is given by

$$
H(x, y)=\int R(x, y) p(x, y) d y+h(x)
$$

where $h(x)$ is chosen to satisfy $\frac{\partial H}{\partial x}=-R q$. Then

$$
\dot{x}=R p=\frac{\partial H}{\partial y}, \quad \dot{y}=R q=-\frac{\partial H}{\partial x} .
$$

In order for this function $H$ to be well defined the open set $U$ must be simply connected.

Conversely, given a first integral $H$ of system (1) we can always find an integrating factor $R$ for which (5) holds.

Let $f \in \mathbf{C}[x, y]$. The algebraic curve $f(x, y)=0$ is an invariant algebraic curve of the polynomial system (1) if for some polynomial $k \in \mathbf{C}[x, y]$ we have

$$
X f=p \frac{\partial f}{\partial x}+q \frac{\partial f}{\partial y}=k f .
$$

The polynomial $k$ is called the cofactor of the invariant algebraic curve $f=0$. We note that since the polynomial system has degree $m$, then any cofactor has at most degree $m-1$.

A (multi-valued) function of the form

$$
\left(\prod_{i=1}^{p} f_{i}^{\lambda_{i}}\right) \exp \left(\frac{g}{h}\right)
$$

where $f_{i}, g$ and $h$ are polynomials and the $\lambda_{i}$ complex numbers, is called a Darbouxian function.

\section{Proof of The Theorem}

We first prove statement (a). We shall use the following result known by Poincaré 16]: If a polynomial system (1) has a rational first integral, then the eigenvalues $\lambda_{1}$ and $\lambda_{2}$ associated to any singular point of the system must be resonant in the following sense: there exist nonnegative integers $m_{1}$ and $m_{2}$ with $m_{1}+m_{2} \geq 1$ such that $m_{1} \lambda_{1}+m_{2} \lambda_{2}=0$. For a proof see, for example, 9 or 11 . Now it is easy to check that the origin of system (2) is a singular point whose ratio of eigenvalues is $1-c$. Since $c$ is irrational, the system can have no rational first integral.

System (2) has the invariant solutions $x=0$ and $x=1$ together with the following two explicit solutions:

$$
\begin{aligned}
& f_{1}=\left(y-\frac{a b}{c} x\right) F_{1}+x(1-x) F_{1}^{\prime}=0 \\
& f_{2}=\left(c-1-y+\left(1-c+\frac{a b}{c}\right) x\right) F_{2}-x(1-x) F_{2}^{\prime}=0
\end{aligned}
$$


where $F_{1}=F(a, b ; c ; x), F_{2}=F(1+a-c, 1+b-c ; 2-c ; x)$, and $F(a, b ; c ; x)$ is the hypergeometric function

$$
F(a, b ; c ; x)=\sum_{k=0}^{\infty} \frac{(a)_{k}(b)_{k}}{(c)_{k}} \frac{x^{k}}{k !} .
$$

Here we have used the notation

$$
(a)_{k}= \begin{cases}1 & \text { if } k=0, \\ a(a+1)(a+2) \cdots(a+k-1) & \text { if } k>0 .\end{cases}
$$

The hypergeometric function $F(a, b ; c ; x)$ is a solution of the hypergeometric differential equation

$$
x(1-x) y^{\prime \prime}+[c-(a+b+1) x] y^{\prime}-a b y=0,
$$

and so $F$ and $F^{\prime}$ can have a common zero only at $x=1$.

If we take $a=1-k, b \leq a$ and $c>0$ irrational, with $k$ a positive integer, then $F(a, b ; c ; x)$ is a polynomial of degree $k-1$, with all coefficients positive. Hence $F(1)>0$, and so $F$ and $F^{\prime}$ can have no common roots at all. Thus $f_{1}=0$ defines an irreducible algebraic curve of degree $k$, which proves statement (b).

Finally, after some calculation it can be shown that

$$
H=\frac{x^{c-1} f_{1}}{f_{2}}
$$

is a first integral of the system, with (reciprocal) integrating factor

$$
x^{c} f_{1}^{2}(1-x)^{a+b+1-c}
$$

which proves statement (c).

\section{ACKNOWLEDGEMENTS}

The first author thanks the Universitat Autònoma de Barcelona for its hospitality at the begining of 2000 . The second author was partially supported by a DGICYT grant number PB96-1153 and by a CICYT grant number 1999SGR 00399.

\section{REFERENCES}

[1] A. Campillo and M.M. Carnicer, Proximity inequalities and bounds for the degree of invariant curves by foliations of $\mathbf{P}_{\mathbf{C}}^{2}$, Trans. Amer. Math. Soc. 349 (1997), 2211-2228. MR 97h:32051

[2] M. M. Carnicer, The Poincaré problem in the nondicritical case, Annals of Math. 140 (1994), 289-294. MR 95k:32031

[3] C. Christopher, Invariant algebraic curves and conditions for a centre, Proc. Royal Soc. Edinburgh 124A (1994), 1209-1229. MR 95m:34052

[4] D. Cerveau and A. Lins Neto, Holomorphic foliations in CP(2) having an invariant algebraic curve, Ann. Inst. Fourier 41 (1991), 883-903. MR 93b:32050

[5] C. Christopher and J. Llibre, Integrability via Invariant Algebraic Curves for Planar Polynomial Differential Systems, Annals of Differential Equations 16 (2000), 5-19. MR 2001g:34001

[6] C. Christopher, P. Mardešić and C. Rousseau, Normalizable, integrable and linearizable saddle points in complex quadratic systems in $\mathbf{C}^{2}$, Preprint, 2000.

[7] G. Darboux, Mémoire sur les équations différentielles algébrique du premier ordre et du premier degré (Mélanges), Bull. Sci. Math. 2ème série, 2 (1878), 60-96; 123-144; 151-200.

[8] J. P. Françoise, A. Fronville and H. Zoladek, Solution of the 1:-2 resonant center problem in the quadratic case, Fundamenta Mathematicae 157 (1998), 191-207. MR 99g:34063

[9] S. D. Furta, On non-integrability of general systems of differential equations, Z. angew Math. Phys. 47 (1996), 112-131. MR 97f:34003

[10] J .P. Jouanolou, Equations de Pfaff algébriques, Lectures Notes in Mathematics 708, Springer-Verlag, 1979. MR 81k:14008 
[11] Weigu Li, J. Llibre and Xiang Zhang, Planar analytic vector fields with generalized rational first integrals, submitted to Bulletin des sciences mathématiques.

[12] A. Lins Neto, Some examples for Poincaré problem and a question of Brunella, preprint.

[13] Yiu-Kwong Man and M. A. H. Maccallum, A Rational Approach to the Prelle-Singer Algorithm, Journal of Symbolic Computation 24 (1997), 31-43. MR 98g:12009

[14] J. Moulin-Ollagnier, About a conjecture on quadratic vector fields, preprint, 2000.

[15] J. M. Pearson, N. G. Lloyd and C. J. Christopher, Algorithmic derivation of centre conditions, SIAM Review 38 no.4 (1996), 619-636. MR 98c:34050

[16] H. Poincaré, Sur l'intégration des équations différentielles du premier ordre et du premier degré I and II, Rendiconti del circolo matematico di Palermo 5 (1891), 161-191; 11 (1897), 193-239.

[17] M. J. Prelle and M. F. Singer, Elementary first integrals of differential equations, Trans. Amer. Math. Soc. 279 (1983), 215-229. MR 85d:12008

[18] M. F. Singer, Liouvillian first integrals of differential equations, Trans. Amer. Math. Soc. 333 (1992), 673-688. MR 92m:12014

School of Mathematics and Statistics, University of Plymouth, Plymouth, Devon PL4 8AA, United Kingdom

E-mail address: cchristopher@plymouth.ac.uk

Departament de Matemàtiques, Universitat Autònoma de Barcelona, 08193 Bellaterra, Barcelona, Spain

E-mail address: jllibre@mat.uab.es 